\title{
Ética Informática en la Sociedad de la Información
}

\author{
Silva, Neif* \\ Espina, Jane ${ }^{\star *}$
}

\section{Resumen}

El propósito de este trabajo es realizar un análisis crítico de las conformidades y desavenencias encontradas en estudios anteriores sobre los cambios producidos por la utilización de las Tecnologías de Información y Comunicación en la sociedad actual. En esta investigación de tipo bibliográfica y documental, los planteamientos realizados forman parte de una revisión teórica de puntos de vista de expertos en el ámbito mundial, se indican los principales rasgos que caracterizan los hallazgos sobre problemas éticos en el área Informática. Las conclusiones destacan tanto las debilidades sobre la perspectiva individualista, que se apoya en la concepción de quien adquiere mayor poder es solo aquel que manipula la tecnología; como la valoración de las potencialidades que existen en la concepción global relativa a la consideración de las tecnologías informáticas como herramientas oportunas para minimizar la complejidad con la que los valores éticos y morales se han tergiversado. Con base en la producción acelerada de nuevas tecnologías informáticas nacidas de la llamada Sociedad de la Información y del Conocimiento, que han provocado serios problemas de carácter éticomoralistas en detrimento de la gerencia del conocimiento y la creatividad del individuo; este artículo evidencia algunas sugerencias en beneficio de la utilización responsable de las Tecnologías de Información y Comunicación que permitirán motorizar la búsqueda de soluciones para lograr el bienestar social de la comunidad y de las organizaciones en general.

Palabras clave: Ética informática, tecnologías de información y comunicación, problemas éticos y morales, sociedad de la información.

\section{Recibido: 06-06-08. Aceptado: 06-10-05}

* Docente Investigador, Centro de Estadística e Investigaciones de Operaciones de la Facultad de Ciencias Económicas y Sociales de la Universidad del Zulia. Ingeniero en Computación. M.Sc. en Planificación y Gerencia de C\&T. Doctor en Ciencias Gerenciales.

E-mail:nsilva@luz.edu.ve

** Docente Investigadora, División de Postgrado e Instituto de Investigaciones de la Facultad de Arquitectura y Diseño de la Universidad del Zulia. Arquitecto. M.Sc. en Arquitectura, Mención Computación en Arquitectura. Doctora en Ciencias Gerenciales.

E-mail: jacky@convergence.com.ve 


\section{Informational Ethics in the Information Society}

\section{Abstract}

The purpose of this paper is to make a critical analysis of the conformities and disagreements found in previous studies on the changes produced by the use of information technologies in present-day society. In this bibliographical and documentary type research, the proposals made form part of the theoretical review of points of view of experts on a world level and the principal findings that characterize their findings as to ethical problems in the information area. The conclusions emphasize the weaknesses in the individualistic perspective that supports the conception that he who acquires greater power is he who manipulates the technology, and the valorization that the potential that exists in the global conception related to the consideration of information technologies as tools to minimize the complexity with which ethical and moral values are based has been twisted. Based on the accelerated production of new information technologies generated by the so-called Information and Knowledge Society, serious problems of an ethicalmoral nature have been provoked in detriment to knowledge management and individual creativity. This article offers some suggestions in order to benefit the responsible utilization of Information and Communication Technologies that motorizes the search for solutions in the achievement of social well-being in the community and in organizations in general.

Key words: Informational ethics, information technologies, ethical and moral struggles, information society.

\section{Introducción}

Este artículo es el resultado de una investigación bibliográfica y documental con sentido crítico, basada en teorías relacionadas con el componente ético y las Tecnologías de Información y Comunicación (TI), cuyo propósito es analizar las conformidades y desavenencias encontradas en estudios anteriores partiendo de los rasgos estructurales que caracterizan la ética informática. En este trabajo se consideran hallazgos en Norteamérica, Europa, Latinoamérica y el Caribe, comprende un análisis conceptual de la ética, la informática, la ética informática y una breve reseña sobre la ética de la información. Además, se presenta una revisión sobre problemas éticos en la utilización de las Tecnologías de Información y Comunicación. Así como, una diserta- ción sobre los códigos deontológicos de la tecnología informática creados por la Sociedad de la Información y del Conocimiento (SIC), finalmente se plantean lineamientos estratégicos para el uso responsable de éstas tecnologías.

Al respecto, el impacto de las Nuevas Tecnologías de Información y Comunicación en la conducta de los seres humanos ha sido estudiado, en la sociedad y en las organizaciones, así como también los problemas y dilemas éticos que se producen, y cómo afectan las relaciones sociales, los valores morales y éticos, normas y aptitudes.

Estas tecnologías proponen definitivamente nuevos estilos de vida y formas de pensar; plantean nuevos paradigmas de relaciones entre los individuos, de allí el surgimiento de los problemas éticos en el uso de éstas y la necesidad de realizar 
estudios que proporcionen soluciones para lograr el bienestar social y la preservación de una cultura informática en las organizaciones.

Los cambios en los aspectos sociales, culturales, políticos y económicos de la sociedad actual generados por los nuevos paradigmas y el avance vertiginoso de las tecnologías de información y comunicación, han producido modificaciones en las características morales y éticas en el uso de las computadoras, programas, información y otros aspectos de índole tecnológico, los cuales a su vez han creado dilemas éticos para los usuarios, los profesionales informáticos y la cultura informática en las organizaciones.

Es importante destacar, que la Cultura Informática en sí, no es el tema central de este artículo, sin embargo guarda cierta relación con las implicaciones éticas que se evidencian en la utilización de las TIC en las organizaciones.

Las conclusiones de este trabajo permitieron formular lineamientos estratégicos para el uso responsable de las TIC por parte de los usuarios, profesionales y las organizaciones, con el fin de promover a nivel gerencial, un análisis del componente ético para la toma de decisiones, y así minimizar el impacto generado por éstas sobre los valores morales y éticos de los individuos y de las organizaciones. Por su parte, el análisis sobre la ética informática posibilitará la solución de los problemas de índole moral y ético que se puedan presentar en el manejo de las TIC en la organización.

\section{2. Ética informática}

En la actualidad los cambios generados por los avances tecnológicos, afectan el comportamiento y las acciones del individuo, conduciendo al planteamiento de nuevas normas o disciplinas para solucionar y dar respuestas a los problemas generados por las Tecnologías de Información y Comunicación. En los últimos años se ha discutido sobre la Ética Informática (CISCI $2006^{1}$ / ETICOMP $2005^{2}$ / IMPACS $^{3}$ / $\left.\mathrm{CNTI}^{4}\right)$, cuáles son sus orígenes, y porqué se hace necesario su estudio. Para entender convenientemente la Ética Informática, es imprescindible conceptualizar individualmente los términos ética e informática.

La ética es entendida por Valls (2000), como una disciplina filosófica definida en principios directivos para orientar a las personas en la concepción de la vida, el hombre, la moral, los juicios, y los hechos; que reflexiona de forma sistemática y metódica sobre el sentido, validez y licitud de los actos humanos individuales y sociales en la historia, también, se ocupa de la fundamentación racional del comportamiento moral del hombre y de encontrar las convergencias axiológicas racionalmente justificables para todo ser humano.

15 a Conferencia Iberoamericana en Sistemas, Cibernética e Informática.

2 Empresarios de Tecnologías de Información y la Comunicación.

3 Information Management Policies Assessment for City Transportation Systems.

4 Centro Nacional de Tecnologías de Información. 
Asimismo, Cortina (1998) la define como "un tipo de saber de los que pretende orientar la acción humana en un sentido racional; es decir, pretende que obremos racionalmente; la ética es esencialmente un saber para actuar de un modo racional". Según Brown (1992), el propósito de la ética no es que la gente sea ética, sino capaz de tomar las mejores decisiones para el bienestar individual y de la sociedad, y que ésta sea el proceso de decidir lo que corresponde hacerse en las organizaciones, ya que éstas son comunidades morales y agentes morales.

La sociedad actual, requiere sujetos responsables de sus acciones, proyectos y realizaciones, necesita una finalidad, sólo desde un contexto semejante tiene sentido la ética, por ende establece fundamentos racionales, normas de comportamiento moral. Los individuos, la sociedad y las instituciones deben combatir contra el hundimiento de la moral, la crisis de los valores, para lograr mayores beneficios un mundo mejor, capaz de enfrentar los dilemas éticos.

Por otra parte, la informática, en este trabajo se entiende como "la ciencia que estudia el fenómeno de la información, los sistemas de información y el procesamiento, transferencia y utilización de la información, mediante computadoras y las telecomunicaciones como herramientas para el beneficio de la humanidad" Hajna (1989:86).

Este concepto se complementa con la definición obtenida de un estudio realizado por Silva (2001), las Tecnologías de Información y Comunicación, son un conjunto de herramientas tecnológicas que se apoyan en la informática: tecnología blanda (software) y tecnología dura (hardware), para la ejecución automatizada de procesos, con criterios de rapidez y precisión; de los cuales se derivan productos que sirven de canales de comunicación para el soporte, almacenamiento, procesamiento y transmisión digitalizados de la información.

El mismo autor señala en un trabajo reciente Silva (2006), que la informática debe entenderse ahora como la ciencia del tratamiento racional de la información a través del uso del computador; considerado éste como el soporte de los conocimientos y de las comunicaciones humanas. La informática está conformada por una serie de técnicas, métodos y máquinas; conocidas como las Tecnologías de Información y Comunicación (TIC's), cuyos beneficios apuntan al tratamiento lógico y automático de la información

Por otra parte, Cornella (1994) citando a Matchand (1980), afirma que las TIC's constituyen "un verdadero rol catalítico, capacitador en la transformación de la sociedad industrial en la sociedad de la información", y que "el verdadero objetivo de las TIC's debe ser el aprovechamiento estratégico de la información para la toma de decisiones de carácter estratégico de la información", por lo que éstas deben ser incorporadas a las organizaciones, para mejorar su competitividad, eficacia y eficiencia en el ámbito empresarial.

Asimismo, Castells (1999:56) señala que las TIC's son "el conjunto convergente de tecnologías de la microelectrónica, la informática, las telecomunicaciones, televisión, radio, ingeniería genética y sus aplicaciones, optoelectrónica, los cuales se utilizan para almacenar, procesar y comunicar la información". 
Finalmente, una posición consensuada puede ser considerada para delinear más aún el concepto de las TIC's. Según OCDE (2002), los países miembros de la Organización para la Cooperación y el Desarrollo Económico, basados en la "International Standard Industrial Classification" (ISIC), el sector de las TIC's es una combinación de actividades de manufactura y servicios para la captación, transmisión y despliegue de información y datos de manera electrónica.

Partiendo de estas afirmaciones, es evidente que en la nueva era de la sociedad del conocimiento, la información y las comunicaciones, las TIC's son factores claves de éxito en los procesos de producción y creación de riqueza. En nuestro país las TIC's cumplen un papel determinante en esta nueva sociedad, por lo que los ciudadanos, y los gobiernos en particular, deben entender y aprovechar los beneficios potenciales que ofrecen estas tecnologías a la sociedad actual.

Las naciones que van a la vanguardia del desarrollo, han comprendido las ventajas competitivas y comparativas de estos elementos tecnológicos, por lo que han tratando de organizar el contexto tecnológico para incrementar su productividad y por ende su bienestar social. Es por ello que han concebido la necesidad de incorporar las TIC's a las principales políticas y programas de desarrollo de sus naciones, como instrumentos de apoyo a la consecución de amplios objetivos nacionales, tanto sociales como económicos.

Los índices relativamente recientes que pueden observarse en la Tabla 1 , sobre la cantidad y porcentaje de usuarios TIC's en el mundo, muestran un mejor comportamiento; los datos dejan en evidencia que las TIC's han venido penetrando cada vez más nuestras sociedades, están cambiando el mundo y continuarán haciéndolo en un futuro.

De allí lo importante de una nueva ética con responsabilidad social que sirva de de guía para rescatar los valores en la utilización de las tecnologías informáticas, es allí donde deben coincidir los be-

\section{Tabla 1 \\ Usuarios TIC en el mundo}

\begin{tabular}{lrccc}
\hline Regiones & $\begin{array}{c}\text { № Habitantes } \\
\text { por Región }\end{array}$ & $\begin{array}{c}\text { Distribución } \\
\text { Poblacional } \\
\text { por Región }\end{array}$ & $\begin{array}{c}\text { Usuarios TIC } \\
\text { por Región }\end{array}$ & $\begin{array}{c}\text { \% de Usuarios } \\
\text { TIC por Región }\end{array}$ \\
\hline África & 805.600 .000 & $13,13 \%$ & 7.942 .800 & $0,99 \%$ \\
América Latina \& Caribe & 560.520 .000 & $9,13 \%$ & 35.441 .200 & $6,32 \%$ \\
Estados Unidos \& Canadá & 319.890 .000 & $5,21 \%$ & 170.217 .000 & $53,21 \%$ \\
Asia & 3.618 .730 .000 & $58,97 \%$ & 201.079 .000 & $5,56 \%$ \\
Europa & 799.630 .000 & $13,03 \%$ & 166.386 .500 & $20,81 \%$ \\
Oceanía & 31.760 .000 & $0,52 \%$ & 10.500 .400 & $33,06 \%$ \\
Total Mundial & $\mathbf{6 . 1 3 6 . 1 3 0 . 0 0 0}$ & $\mathbf{1 0 0 , 0 0 \%}$ & $\mathbf{5 9 1 . 5 6 6 . 9 0 0}$ & $\mathbf{9 , 6 4 \%}$ \\
\hline
\end{tabular}

Fuente: Elaboración Propia (2006) basado en [ITU] (2003). 
neficios potenciales de la ética y la informática. Es necesario entonces plantear una nueva forma de gerenciar incorporando las nuevas tecnologías de información y comunicación con base en el negocio, de manera que, estas permitan mejorar la ejecución de los procesos básicos del negocio y el desempeño de las funciones gerenciales para manejar eficientemente los costos que se produzcan en servicios de información y así fomentar al mismo tiempo la credibilidad de estos servicios por parte de los clientes potenciales (Mc Leod, 2000).

Es importante señalar que al hablar de la ética informática, se habla de una ética global emergente y de todos los aspectos de la vida del ser humano. Partiendo de este principio, se plantea el tratado sobre los deberes y derechos de los profesionales de la informática. Según un planteamiento que hiciera Joyanes (1997), afirmando que la ética informática adquiriría un carácter global, aún se mantiene vigente, puesto que las tecnologías informáticas como las redes de computadoras y el ciberespacio han producido una revolución informática donde el componente ético ha sido fundamental para la totalidad de las acciones y relaciones del ser humano en la era de la post-Información.

La relación existente entre la ética y la informática es debido al impacto de tecnologías de información y comunicación en la sociedad actual, en la cual surgen aspectos que vinculan ambas disciplinas. Cabe citar por ejemplo, la invasión de la privacidad del usuario en la red Internet, la cual se pone en evidencia a través del envío de correos con publicidad electrónica "spamming"; otras acciones como la copia ilegal de programas de computa- ción, la transferencia de datos de un servidor sin autorización, entre otros. Por lo tanto, los usuarios y profesionales de la informática deben definir sus responsabilidades y tomar las decisiones mas justas para lograr el bienestar social en la utilización de estas tecnologías.

Según Moor (1985) la ética informática se define como la disciplina que identifica, analiza la naturaleza y el impacto social de las tecnologías de la información y la comunicación en los valores humanos y sociales; estos son: salud, riqueza, trabajo, libertad, privacidad, seguridad o la autorrealización personal, democracia, conocimiento, entre otros. Además, involucra la formulación y justificación de políticas para dirigir nuestras acciones, $y$ hacer un uso ético de éstas tecnologías.

Al respecto, es conveniente señalar, que la ética informática es una disciplina emergente dentro de la ética profesional, de importancia vital para la sociedad y las organizaciones actuales. Por su parte, Joyanes (1997:272) la considera como "el análisis de la naturaleza e impacto social de la tecnología informática, la formulación correspondiente y justificación de políticas para su uso ético".

Por otra parte, Del Brutto (2003) la define como la disciplina que analiza los problemas éticos que son creados, transformados o agravados por las personas que utilizan los avances de las tecnologías de la información.

Asimismo, Cortina (1998) afirma que ésta es una vertiente de la "Ética Aplicada" y surge como una necesidad de generar una deontología profesional en el uso de las tecnologías de información y comunicación; basada esta, en códigos de ética que permitan ocupar el vacío 
existente de normas, que orienten su utilización en la resolución de problemas creados por el hombre en la sociedad a través de su uso.

De acuerdo a estos planteamientos es posible afirmar que cada dilema ético es un problema que surge en los profesionales y usuarios de los equipos tecnológicos de computación. En la búsqueda de soluciones adecuadas a cada problema, se genera cada vez una nueva forma de actuar.

Al respecto cabe citar que la sociedad actual se vuelve más vulnerable al mal uso de los computadores por parte de los seres humanos, frecuentemente nos encontramos por ejemplo con el mal funcionamiento de un equipo originado por fallas en los programas de computación y en los sistemas informáticos. Asimismo, cada vez se generan nuevas formas de inserción de virus en los sistemas de cómputo que exigen acciones inmediatas que solucionen los problemas que todo esto ocasiona. La mayoría de estos problemas son producidos por personas mal intencionadas cuyo único fin es llevar a cabo los llamados "delitos informáticos".

Según Joyanes (1997) el desarrollo de la ética aplicada a la informática, es una vía para combatir estos delitos informáticos. Existe la necesidad imperante de restablecer el contexto social, en una sociedad de la información global, compleja y vulnerable. Una de las tareas más importantes de esta ética es plantear la formulación de nuevas normas y leyes que protejan la información privada y los procesos de trabajo, ya que se presentarán situaciones que ameritarán soluciones éticas o profesionales para las organizaciones de esta nueva sociedad.

Es importante entonces, plantear una reflexión sobre la ética informática, ya que esta filosofía se fundamenta en el uso de las TIC's bajo principios morales y éticos. Es necesario además, que se produzcan nuevos códigos de ética; normas personales y sociales aplicables a las ciencias y nuevas profesiones, a la conducta del ser humano y a la organización, todo lo cual permita un uso ético de éstas tecnologías.

Ahora bien, bajo esta perspectiva las tecnologías de información y comunicación posibilitan generación de nuevos paradigmas a través de la renovación de procesos, nuevas formas de organización, nuevos modelos y cambios en la forma de trabajar, todo esto repercute sobre el mundo empresarial.

Este planteamiento pareciera cobrar fuerzas dadas las intenciones emitidas por representantes de cada país a nivel mundial en incrementar las inversiones en nuevas tecnologías de información y comunicación para la asistencia de los procesos académicos, gubernamentales y empresariales de los países de $\mathrm{ALAC}^{5}$ (WSIS $2003,2005^{6}$, EIR $2005^{7}$, OEA $2006^{8}$ ).

6 World Submit for the Information Society. 1ํ Session Geneva 2003. 2ํㅗㅇ Session Tunis 2005.

7 Encuentro Internacional de Rectores. Sevilla-España 2005.

8 Organización de Estados Americanos. Reunión Junio 052006. 
Los cambios producidos por esta revolución tecnológica centrada en la información han generado profundas transformaciones en nuestro modo de pensar, actuar, organizar, producir, consumir y comunicar, de hacer la guerra y el amor.

Tal y como señala Castells (1999), se están cambiando los valores morales y éticos, forjando una cultura informática de la virtualidad real. Estas macrotransformaciones son resultado de la interacción de procesos en la "Era de la Información, informacionalización, globalización, interconexión, construcción de identidad, crisis del patriarcado y del Estado-nación".

Por ello, es importante reconocer que los problemas que se generan en la sociedad de hoy pueden tener solución y que el valor de la ética será capaz de influir e intervenir en la toma de decisiones más adecuadas, que existen criterios éticos y que la ética informática es la ética de la lógica de la vida.

\section{3. Ética de la información}

Según Capurro (2005), la definición de ética de la información se remonta aproximadamente a la década de 1970 cuando los equipos de computación comenzaron a utilizarse en el campo de la información de carácter científico-técnico, surgiendo incógnitas con respecto al almacenamiento y accesibilidad a documentos generados, localizados en bases de datos de tipo bibliográficas. Luego este concepto se amplió con el uso masivo de la Red Internet, por los problemas éticos generados.

En la actualidad la ética de la información comprende las interrogantes éticas relacionadas con el proceso de la di- gitalización, la comunicación de sus resultados y la utilización adecuada de la información generada. La ética de información trata todo lo relacionado con el uso y mal uso de la información, tales como, la propiedad intelectual, acceso a la información libre o restringida, censura, uso de información de las instituciones gubernamentales, la intimidad y confidencialidad, integridad de los datos, flujo internacional de información, entre otros. También, la ética profesional realiza el análisis de cómo se emplean los principios éticos a nuestras decisiones y acciones como profesionales de la información (Fernández, 2000).

En este mismo orden de ideas, la ética de la información puede concebirse como una teoría descriptiva y emancipadora bajo dos perspectivas históricas o sistemáticas, en la primera, analiza las diferentes estructuras y relaciones de poder que establecen la actividad informativa en las diversa culturas y épocas, mientras que en la segunda trata la crítica al proceso de relaciones morales en el campo de la información, albergando los aspectos individuales, colectivos y universales (Capurro, 2005).

Es importante señalar, que esta nueva disciplina, surge en los últimos años como multidisciplinaria del campo filosófico y científico, está dentro de los nuevos desafíos morales y éticos representados por la revolución digital y de la computadora. El uso de ésta ética no será útil para resolver los problemas específicos de la ética informática, pero tratará de mantener los principios morales que guiaran los procedimientos para resolver éstos, ya que las normativas, códigos profesionales de conducta, y las legisla- 
ciones para el uso de los equipos de computadores o de la información, están basadas en una ética filosófica.

Según Floridi (2000) la Ética de la Información, se ocupa de dos aspectos importantes, los cuales se indican a continuación:

- Problemas conceptuales que surgen en la aplicación de preexistir teorías, conceptos, normas tradicionales.

- Defensa del destino teórico en el campo de la moral, la deontología del estado moral de la entidad información, la metodología ética, el papel de la información en el razonamiento moral, la epistemología moral y las decisiones en los contextos dominados por las nuevas tecnologías y la epistémica de la responsabilidad. La pregunta principal, qué es bueno para una entidad informativa y para la infosfera «el ambiente de la información»

Cabe destacar, que el mismo autor señala la existencia de cuatro Leyes Morales de la ética de la información para garantizar el bienestar de cada entidad informativa y la infosfera:

- Nunca se tiene que producir entropía «ausencia de información semántica» en la infosfera.

- Debe prevenirse la entropía en la infosfera.

- Se tiene que eliminar la entropía en la infosfera.

- Se debe garantizar el bienestar, la cantidad, calidad y variedad de la información en la infosfera.

Estas cuatro leyes clarifican las grandes líneas de cómo pueden vivir las personas, como agentes responsables en la Sociedad de la información o postinformación. A continuación se concep- tualizan los principios que según Floridi (2000) adopta la ética de la información:

- La uniformidad: todo se procesa, se tratan los funcionamientos, cambios, acciones y eventos como procesos de información, pero el proceso no será tomado como tal, sino se seleccionará lo más significativo de la actividad.

- La reflexividad de procesos de información: cualquier proceso de información necesariamente genera y es responsable de la información.

- La inevitabilidad de procesos de información: la ausencia de información también es un proceso de información.

- La uniformidad de ser: una entidad es un paquete consistente de información, que puede nombrarse y la ética trata cada entidad como una entidad de información.

- La uniformidad de agencia: un agente es cualquier entidad, capaz de definir fenómenos para la producción de información y que puede afectar la infosfera. No todas las entidades de información son agentes.

- La uniformidad de no ser: no ser, es la ausencia o negación de cualquier información o lo que se denomina entropía de información, esta es una cantidad especifica de desorden, degradación o aleatoriedad en un sistema de energía productiva de información, es el ruido.

- La uniformidad del ambiente: la infosfera es el ambiente constituido por la totalidad de las entidades, incluyendo los agentes, los procesos, sus conveniencias y relaciones.

Además, el ambiente de la información o infosfera, también posee propieda- 
des, según Floridi (2000) son: consistencia, implementabilidad, ocurrencia, persistencia, estabilidad, seguridad, confidencialidad, integridad, exactitud, autenticidad y fiabilidad, entre otras.

Por otra parte, la ética de la información debe satisfacer cuatro propiedades para lograr sus objetivos, éstos son los siguientes:

- Estabilidad: la evolución de la tecnología ha significado una expansión rápida del ciberespacio, la sustitución de actividades realizadas por humanos por computadores o sistemas expertos y el impacto de las tecnologías de información y comunicación directamente es sobre los seres humanos.

- Modularidad: la ética se apoya en el razonamiento modular e incremental, los sistemas de información y los componentes del ciberespacio son productos complejos de ingeniería, por lo tanto, se debe modular éste.

- Rigurosidad: establecimiento de una teoría ética basada en un razonamiento riguroso.

- Entereza: debe haber un lugar en la ética de la información para la codificación de los valores, y permitir un análisis reflexivo para el estudio de casos típicos, ser legítima, excluir la trivialidad, ya que cada declaración se juzga para ser verdad.

Sin embargo, la ética de la información no es la última palabra en materia de moralidad, no proporciona un listado de soluciones a los problemas morales que surgen, pero puede mejorar la perspectiva y es lo más conveniente para la cultura informática de la organización y la sociedad de la post-Información. Esta ética mejora la comprensión de los hechos mo- rales, mantiene el sentido del valor, la rectitud y las equivocaciones en las acciones humanas más intangibles y explicables, no solamente en nuestra vida personal sino también en las organizaciones. Además, proporciona una mejor visión y discernimiento no sólo de los problemas morales en su propio campo especial, sino también, de los fenómenos conceptuales y morales que forman el discurso ético, ampliando la perspectiva a la información y a su espacio lógico.

\section{Problemas éticos en la utilización de las tecnologías informáticas en las organizaciones}

Los problemas éticos han sido estudiados durante muchos años, se han realizado conferencias en el ámbito internacional en los problemas éticos de tecnología de información, interviniendo los Países Bajos, Reino Unido, EEUU, Italia, entre otros. La ética de la información está basada en la fundación filosófica de ética informática, esta ética informática es calificada como una disciplina filosófica, que según Floridi (2000) comparte con otras disciplinas filosóficas la tradición analítica, tres rasgos importantes la caracterizan: es lógicamente argumentativa, con perjuicio para el razonamiento analógico, se conecta con tierra empíricamente, con perjuicio para el análisis de los guiones y por último endosa un problema que resuelve el acercamiento.

La ética informática va de la mano con la revolución de las TI, éstas además de su complejidad, del mal funcionamiento y el mal uso de las mismas, crea un nuevo rango de problemas sociales, éticos y morales. 
Durante la revolución digital, las tecnologías de la información han generado profundas transformaciones radicales, generando problemas de tipo social, no sólo transformando el contexto de los problemas pasados sino que también agrega nuevas dimensiones interesantes a los mismos.

Estos problemas penetran e influyen en casi cada aspecto de nuestra vida, de la sociedad y de la organización, ocurren famosas transgresiones, tales como: el «spamming», que es el uso indebido de la red para hacer publicidad no solicitada, las llamadas cartas cadena, que pueden incluso hacerle perder su acceso a Internet, el «flameo» que no es otra cosa que responder agresivamente los ataques violentos de un remitente, la seguridad, la piratería de la computadora, la copia ilegal de software, la libertad de palabra, las discriminaciones intelectuales y sociales, el vandalismo (crackers), las bromas (hackers), los virus (gusanos, troyanos), la pornografía digital, la venta ilegal de órganos, la desinformación, la experimentación con la identidad ficticia dentro de la realidad virtual, el anonimato, la propiedad intelectual y derechos de propiedad.

En este mismo orden de ideas, los problemas en los sitios de trabajo informatizados, las aplicaciones de las leyes en el ciberespacio, la ética de la computadora profesional y la metodología de códigos profesionales son temas para el estudio de la ética. Surgen entonces problemas en los ambientes de trabajo, códigos de ética profesionales para los consumidores y profesionales de la informática, la ética de la inteligencia artificial y la vida artificial, los problemas éticos de la sociedad de la información son transformados profundamente por la tecnología de la información, esta no solo tiene un valor por el discurso moral y en nuestros conceptos éticos sino también en nuestras experiencias personales, nuestra identidad mental y física.

Los crímenes o actos ilegales o no éticos por medio de la red Internet o cualquier otra forma de enlace computacional son más frecuentes y peligrosos, un ejemplo muy conocido fue el de dos estudiantes de la Universidad de Berkeley, colocaron una petición en la Web en contra del juicio político contra Clinton, cuando fue presidente de los Estados Unidos de Norteamérica, consiguieron un total de 160.000 mil firmas, y se las mandaron al Comité Judicial del Congreso de los Estados Unidos, simultáneamente, un grupo de Virginia reunió 30.000 firmas solicitando enjuiciar en ese mismo periodo al Presidente Bill Clinton.

De la misma forma, pueden cometerse delitos por cualquiera de los medios ya conocidos a través de Internet, con la diferencia de que la red añade una mayor facilidad y difusión, y especialmente una internacionalización del delito que ha sorprendido a todo tipo de juristas en todo el mundo. Nuevos conflictos surgen para los legisladores, cómo éstos pueden aplicar la ley y juzgar esos problemas. Otro de los ejemplos, es el de una página web que reside en un servidor en Corea, se insultan a los gobernantes, o empresarios españoles, tales delitos no pueden perseguirse en algunos países, pues los usuarios de Internet libremente acuden electrónicamente a Corea. Las situaciones antes señaladas, están obligando a los muchos países del mundo a modificar sus legislaciones y a establecer tratados 
internacionales, para juzgar esos delincuentes informáticos.

Cabe destacar, que este tipo de guerra se basa en comunicaciones sofisticadas, en la incorporación de inteligencia, el acceso al espacio, a lo que muchos llaman el "cuarto espacio o ciberespacio", $y$ en circuitos de decisiones en tiempo real, este espacio es producto de los desarrollos en el área de las tecnologías de información y de la comunicación.

Esta guerra es de tipo informativa, es simplemente el uso de la información para alcanzar sus objetivos, la información por si misma es poder, de allí la celebre frase "tener la información, es tener el poder", ese conflicto desarrollado a través de los medios informativos y de comunicación, utilizar redes de interconexión a nivel de las sociedades o entre naciones, además no sólo se refiere a lo anterior, sino también la influencia que ésta tiene sobre los seres humanos y en la toma de decisiones, se puede generar un desequilibrio entre el contrincante y el oponente, es decir, el blanco es la mente humana y la voluntad del adversario, de sus sistemas de conocimientos y creencias.

Asimismo, el campo de batalla de esta guerra es el "espacio cibernético", en este tipo de guerra un nuevo factor de vital importancia es la "infosfera", que es el ambiente de la información, cuales son sus herramientas, las armas informativas, las cuales son extremadamente poderosas y que en el caso de organizaciones pueden llevarlas al caos, puede también atacar los sistemas de información de las empresas, financieros, telecomunicaciones y las infraestructuras de las organizaciones, un ejemplo de ellas son los virus informáticos, impulsos electro- magnéticos, entre otros . Las personas o empresas que utilizan este tipo de guerra, lo pueden hacer inconscientemente o deliberadamente, a veces analizando los riesgos morales y éticos que puede causar.

Por lo tanto, la utilización de las tecnologías de información y la comunicación de punta, pueden ser aplicadas en la guerra de la información a nivel estratégico, desarrollando una visión y una capacidad real, realizando por ejemplo, simulaciones de la realidad, a través de la combinación de técnicas de computación, ya que los objetivos de esta guerra son apoderarse de las "decisiones que están en la mente del oponente, y el espacio de la batalla en la mente humana también es la zona de ilusión" (Stein, 2000:4).

Los sistemas de información conectados a las autopistas de información son considerados cada día más como armas estratégicas, las cuales son imprescindibles en la preparación del camino a las organizaciones para que éstas penetren el mercado de su competencia y pueden mantenerse en él, no como organizaciones de supervivencia sino como organizaciones vitales. Por ende las organizaciones deben estar preparadas para la guerra de información, los autores Álvarez y Rodríguez (1998) afirman que el actual equilibrio de poder como también la balanza competitiva puede ser modificados en dependencia de quien tome la delantera en la guerra de información.

\section{Códigos deontológicos de la tecnología informática}

Existen códigos y normas éticas para el uso de las tecnologías de informa- 
ción y comunicación, especialmente los computadores, los cuales dependen de la organización, empresa o institución. Las asociaciones de profesionales de informática y algunas empresas relacionadas con la informática han desarrollado códigos de conducta profesional $\left(\mathrm{ACM}^{9}\right.$, $\mathrm{IEEE}^{10}, \mathrm{BCS}^{11}, \mathrm{IFIP}^{12}$ ). Estos códigos tienen distintas funciones:

- El usuario de los recursos computacionales o de comunicación tiene la responsabilidad de usarlos de manera ética, profesional y con apego a la ley.

- Las normas éticas para una profesión, exigen al profesional no solo la responsabilidad sobre los aspectos técnicos del producto, sino también de las consecuencias económicas, sociológicas y culturales del mismo.

- La velocidad del desarrollo de las tecnologías de la información hacen que los códigos o normas éticas sirvan de ayuda a los cuerpos legislativos, administrativos y judiciales; como un instrumento flexible o como suplemento a las medidas legales y políticas que en general se aprueban en un período de tiempo muy largo y en desfase con el avance de las TIC's.

- Las normas éticas sirven para hacer al usuario consciente de los problemas y estimula un debate para designar responsabilidades.

- Estas normas tienen una función sociológica ya que dan una identidad a los informáticos como grupo que piensa de una determinada manera; es símbolo de sus estatus profesional y parte de su definición como profesionales.

- Estas normas sirven también como fuente de evaluación pública de una profesión y son una llamada a la responsabilidad que permiten que la sociedad sepa qué pasa en esa profesión; aumenta la reputación del profesional y la confianza del público.

- Estas normas permiten armonizar legislaciones o criterios divergentes existentes (o ausentes, en su caso) en los países individuales.

Sin embargo, la crítica que se hace a estas asociaciones, es que han hecho poco por hacerlos cumplir, por imponer sanciones si no se cumplen o por comprobar si se aplican o si son relevantes o pertinentes. De hecho, hay códigos que no son conocidos por los miembros de sus profesiones ni por sus clientes. Parker (1990) denuncia que tampoco se reinterpretan, es decir, que exceptuando las situaciones más obvias, que son a las que hacen referencia estos códigos, no se sabe casi nada de la eticidad de la mayoría de las acciones en las que se mueven los informáticos. En general, también suelen faltar las medidas disciplinares, necesarias cuando las actividades de un miembro están en conflicto con la letra o el espíritu del código. También, se critica

\section{Association for Computing Machinery.}

10 The Institute of Electrical and Electronics Engineers.

11 Brithish Computer Society.

12 Internacional Federation for Information Processing. 
que muchos códigos son el fruto del pensamiento tecnológico de los países desarrollados que no tienen en cuenta diferencias en valores sociales y culturales. Incluso hay asociaciones feministas que opinan que los códigos éticos de las asociaciones de informáticos reflejan valores denominados "masculinos" pues son códigos "creados por profesionales varones para técnicos varones".

En general, los códigos no atienden a los grandes temas éticos de justicia a los que nos enfrentamos en nuestro tiempo: desigualdad económica, desempleo, pobreza, racismo, opresión del tercer mundo. La relación de estos problemas con las tecnologías de la información no es directa, ni unívoca ni de una forma en la que haya un consenso global pero al menos sí se admite que los ordenadores $y$ las telecomunicaciones, al ser ya parte de nuestra vida colectiva, pueden y deben aportar algo en estos problemas .

Es importante destacar, que las asociaciones de profesionales de informáticos planteen nuevos códigos de ética para obligar de algún la manera de actuar. Esto quiere decir que en esta sociedad actual, llamada tecnocrática, los técnicos están reflexionando sobre las consecuencias de su trabajo. Además, en la mayoría de los casos, son los informáticos los que tratan en profundidad la naturaleza de los sistemas informáticos, los sistemas de seguridad, los posibles daños por un mal uso del sistema y la verdadera intención de sus usuarios. Por ello, para evitar confusiones sobre la relación entre la profesión y la sociedad hay que responder convenientemente a qué fin o bien sirve un informático, cómo es el proceso de toma de decisiones en la relacio- nes entre tu profesión y tus obligaciones en la sociedad.

Los códigos son un paso en la concienciación de las sociedades y organizaciones que quieren mejorar situaciones en las que los impactos sociales del desarrollo tecnológico no se tienen en cuenta. No tienen que duplicar lo que ya existe en la ley. La ley trata de la legalidad de las prácticas sociales, es normativa por definición y se impone con sanciones. Los códigos, en cambio, tratan del comportamiento según principios éticos, su normatividad es no más mostrar una declaración de intenciones sobre la "misión" de una institución y la coerción real con que se imponen es pequeña, aunque en algunos casos se incluyen expulsiones de la asociación en cuestión. La ley es el acercamiento de más poder normativo y asigna con claridad los derechos, responsabilidades y deberes de cada uno.

\section{Influencia de las tecnologías de información y comunicación en los individuos y en la gerencia}

Este mundo digital o cibermundo ha logrado que los seres humanos hayan perdido poco a poco, su contacto entre sí. Joyanes (1997) afirma que la transición hacia el mundo digital ha afectado al individuo, $y$ lo ha convertido en un ser con un carácter más deshumanizador y superficial, y lo ha obligado en alguna medida a incorporarse al ciberespacio que sustituye el mundo real por uno virtual, tal como se puede observa en el siguiente gráfico en algunos sectores del mundo la incorporación a Internet ha sido mayor o menor por distintas causas. América Latina contaba para finales del año 2002 con 35 
millones de usuarios, casi el $7 \%$ de la población total de esa región. Al respecto, se presume que el nivel de transformación ${ }^{13}$ las relaciones entre las personas, de cada zona es proporcional al nivel de penetración de usuarios.

Penetración de Usuarios de Internet en la población total

\begin{tabular}{|c|c|c|}
\hline Regiones & Usuarios & $\begin{array}{c}\text { Penetración } \\
\text { Internet }\end{array}$ \\
\hline África & 7.942 .800 & $0.99 \%$ \\
\hline $\begin{array}{l}\text { América Latina } \\
\& \text { Caribe }\end{array}$ & 35.441 .200 & $6.32 \%$ \\
\hline $\begin{array}{l}\text { Estados Unidos } \\
\text { \& Canadá }\end{array}$ & 170.217 .000 & $53.21 \%$ \\
\hline Asia & 201.079.000 & $5.56 \%$ \\
\hline Europa & 166.386 .500 & $20.81 \%$ \\
\hline Oceanía & 10.500 .400 & $33.06 \%$ \\
\hline Total Mundial $^{\star}$ & 591.566 .900 & $9.64 \%$ \\
\hline \multicolumn{3}{|c|}{$\begin{array}{l}\text { * El porcentaje de penetración alcanzado a nivel } \\
\text { mundial se calcula en referencia a la población total } \\
\text { mundial. } \\
\text { Fuente: Elaboración propia (2006). Basado en [ITU] } \\
(2003) \text {. }\end{array}$} \\
\hline
\end{tabular}

Como puede observarse, aún cuando la diferencia entre los índices porcentuales de ALAC en referencia a los países desarrollados es considerablemente alta, los cambios tecnológicos generados han modificado irremediablemente el carácter moral del entorno y por ende el de los individuos, todo lo cual ha generado a su vez dilemas éticos en los usuarios de las tecnologías; muy por en- cima de las afirmaciones de autores sobre las tecnologías de información y comunicación, que son utilizadas en organizaciones para la automatización de los procesos, mejoran la vida laboral y por ende la calidad de vida del individuo: Sin embargo, en contra parte está automatización informatización puede generar problemas a los individuos, tales como el estrés, generando consecuencias graves sobre la salud de los mismos.

Joyanes (1997) mencionó en su libro "Cybersociety" que los impactos más sobresalientes la nueva cultura en esta sociedad digital eran: enriquecimiento e igualdad de la cultura, acceso al arte, intercambio y comunicación de tipo electrónica, formación continua; pero que sucederían cosas como: reducción de empleos, erotismo, sexo y pornografía virtual, la explosión del ocio, el hogar digital y algunos aspectos no señalados por el autor y que están ocurriendo en la actualidad, como lo es el tráfico de órganos y armas, entre otros.

Por otra parte, los valores más importantes que se ven afectados por los efectos sociales de las tecnologías de información y comunicación son los siguientes: la autonomía individual, el acceso a beneficios y oportunidades para todos por igual, sin restricciones con libertades en una sociedad más justa, sin problemas raciales. En este sentido, cómo la ética informática puede resolver el problema de los llamados "laggards" ${ }^{14 ",}$ de que no todas las personas ni las orga-

13 Entendido generalmente, en este contexto, como el reemplazo del contacto humano por el contacto vía Internet.

14 Individuos que no han usado las TIC, ni Internet y no están interesados en hacerlo. 
nizaciones posean computadoras, ni puedan acceder a las sistemas más sencillos de procesamiento de datos, es decir, no tienen acceso a la información en el sentido estricto, a las nuevas tecnologías. En los países de ALAC, son muchos los que poseen escasas posibilidades de acceder a las TIC, evidencia de esto es el cuadro que se presenta a continuación:

Penetración de Usuarios de Internet en América Latina

\begin{tabular}{|l|c|c|}
\hline Chile & 3.102 .200 & $20.14 \%$ \\
\hline Colombia & 1.982 .000 & $4.57 \%$ \\
\hline Puerto Rico & 600.000 & $15.63 \%$ \\
\hline Panamá & 120.000 & $4.13 \%$ \\
\hline Uruguay & 400.000 & $11.90 \%$ \\
\hline Ecuador & 503.300 & $3.88 \%$ \\
\hline Argentina & 4.100 .000 & $11.20 \%$ \\
\hline Honduras & 200.000 & $2.97 \%$ \\
\hline Costa Rica & 384.000 & $9.33 \%$ \\
\hline Bolivia & 180.000 & $2.17 \%$ \\
\hline Brasil & 14.300 .000 & $8.22 \%$ \\
\hline $\begin{array}{l}\text { República } \\
\text { Dominicana }\end{array}$ & 186.000 & $2.14 \%$ \\
\hline Perú & 2.000 .000 & $7.66 \%$ \\
\hline Paraguay & 100.000 & $1.73 \%$ \\
\hline Venezuela & 1.274 .400 & $5.03 \%$ \\
\hline Guatemala & 200.000 & $1.71 \%$ \\
\hline El Salvador & 300.000 & $4.64 \%$ \\
\hline Nicaragua & 90.000 & $1.67 \%$ \\
\hline México & 4.663 .400 & $4.57 \%$ \\
\hline Cuba & 120.000 & $1.06 \%$ \\
\hline $\begin{array}{l}\text { Fuente: Unión Internacional de } \\
\text { nes [ITU] (2003). }\end{array}$ & \\
\hline
\end{tabular}

15 Información referencial de Tendencias Digitales, C.A.
En referencia a esto se puede señalar que según una reciente investigación, Venezuela en poco menos de 2 años ha logrado alcanzar casi los 4.500 .000 de usuarios conectados a Internet lo cual representa un $11,66 \%{ }^{15}$. Es definitivamente comprometedor declarar sin duda alguna que la ética aplicada al área informática pueda resolver todos estos problemas que se producen en el área informática por el empleo de las TIC. Es muy probable que el componente ético pueda orientar y motivar al buen uso de estas tecnologías, en lo que los usuarios deben hacer, cumplir con el debe ser, en la escala de valores.

La revolución tecnológica ha originado cambios sociales en las personas, en valores de los ciudadanos, en las relaciones, en los grupos, en las instituciones, organizaciones y por ende en la sociedad; ha reorganizado nuestras vidas, lugares de trabajo, hogares, privacidad, identidad, la conducta moral del hombre en la sociedad de la post-información.

Estos cambios causados en las organizaciones de la nueva Sociedad de la post-información, ha permitido que la información, la comunicación y las tecnologías hayan pasado a ser recursos de vital importancia dentro de la empresa. En este sentido, la información no debe ser considerada unidimensional sino transdimensional, puesto que el individuo de la era de la post-información, trabaja ahora con la información como valor añadido, como centro de información del poder. 
Al respecto es pertinente mencionar que actualmente existen muchas organizaciones que aunque disponen de novedosa tecnología vanguardista, los individuos que la dirigen aún no tienen una cultura informática instaurada en los procesos organizacionales y mucho menos en sus empleados.

De allí, que en la actualidad, las organizaciones analicen la clave de esta sociedad, el nuevo pensamiento informacional ${ }^{16}$ ó la nueva información para el conocimiento, no sólo en la tecnología, sino también la generación de nuevas estructuras de pensamiento que esta sociedad plantea con base en nuevos esquemas y tendencias post-informacionales, que transforman los valores morales y éticos, las formas del poder, del saber y del hacer.

La preocupación de la gerencia de las organizaciones en esta sociedad dinamizada por estos cambios que han acelerado los procesos organizacionales, generando cambio de ideas, creando dudas e incertidumbre de la razón a través de la interactividad del mundo virtual y autopista de la información debe girar en torno a crear espacios de discusión para combatir estos tiempos inciertos y turbulentos, para enfrentar muchas de las cosas que viene dadas por los cambios tecnológicos.

La organización debe estar preparada para auto dirigirse en este siglo XXI con concepciones actualizadas, gerenciarse en mundos virtuales, con la información, la comunicación y las nuevas tecnologías. Este es uno de los retos de la nueva gerencia, ya que en la actualidad se trabaja con bancas virtuales, empresas globales y virtuales, saber y gerencia virtuales, cambio de los roles, de los procesos de trabajo y de los valores organizacionales.

De esta forma, las Tecnologías de Información y Comunicación como señala Zavarce (1998), pueden servir de ancla para construir estrategias competitivas o también para apoyar estrategias de cambio, establecimiento de nuevos modelos organizacionales que respondan al proceso y manejo del cambio y nuevos principios en su modo de trabajar, este cambio puede ser incremental o paradigmático.

\section{Lineamientos estratégicos gerenciales para el uso responsable de la tecnología informática computacional}

En cuanto a tecnologías se refiere, se deben conservar los niveles de utilización que actualmente se evidencian en la mayoría de las organizaciones, como lo son: los elementos del escritorio digital y las herramientas de comunicación. Sin embargo, es imprescindible prestar atención a los puntos débiles que existen en las organizaciones sobre la base de nuevas tecnologías emergentes que aún no han sido incorporadas. Para lograr un equilibrio y un máximo uso de las tecnologías informáticas, se plantean los siguientes lineamientos: 
- Los medios de producción de información deben ser incorporados, mediante líneas telefónicas que permitan la utilización de la tecnología de buzón de voz.

- La programación de presentaciones en la modalidad de foros o charlas que orienten sobre la utilización efectiva de las TIC's.

- La planificación de dinámicas de grupo para los integrantes de la organización a fin de elevar el nivel de apoyo en la toma de decisiones, y conducirá por consiguiente a aumentar el nivel de divulgación del conocimiento de los códigos éticos para la utilización de la tecnología informática, esto significaría un excelente aporte al crecimiento intelectual individual y del grupo en general.

- Sensibilizar al personal de la organización en el buen uso y aprovechamiento de las tecnologías existentes, a fin de facilitar la incorporación de nuevas tecnologías emergentes.

- Planificar cursos de extensión para el conocimiento de los sistemas de comunicación en tiempo real y los sistemas de análisis y colaboración, que además de posibilitar el aprendizaje de estas herramientas, ayuden a despertar en cada uno de los integrantes de la organización la necesidad de utilizarlas y al mismo tiempo puedan descubrir los beneficios potenciales que estas pueden proveer.

- Evaluar la posibilidad de adoptar una modalidad que pueda ser incorporada al control del personal de la organización, esquema que motivaría a la posible reducción de algunos problemas éticos como: retrasos, inasisten- cias y anormalidades que en general pudieran estar afectando el desempeño laboral y la calidad del servicio de la organización.

- Definir políticas de información, comunicación y divulgación de la utilización de las TIC's a través de una gerencia efectiva de Tecnologías de Información y Comunicación, a fin de orientar su manejo adecuado.

- Promover la formulación de un Programa de Automatización Global (PAG) sobre la base de un Sistema de Gestión de Tecnologías de Información y Comunicación que según Silva (2001), el éxito del mismo depende de la claridad de la gerencia y del componente ético organizacional.

- EI PAG debe promover la adquisición de tecnologías de vanguardia, como las computadoras de bolsillo, interfaces inteligentes, y otros elementos que deben ser incorporados en la organización, y que pueden ser muy útiles como herramientas de apoyo en los procesos. Este programa deberá aprovechar al mismo tiempo, la actual disposición del empresariado a participar en actividades de vinculación, y en consecuencia crear condiciones apropiadas de motivación empresarial para asegurar mejores senderos de viabilidad externa al recurso humano y producción tecnológica.

Las organizaciones en general deberán reforzar la importancia de la presencia del componente ético en sus procesos gerenciales apoyados por la tecnología informática. Los códigos éticos deontológicos deben ser asumidos por los empleados de manera que éstos puedan sensibilizarse para un mejor uso 
de la tecnología informática, deben profundizar el compromiso moral con su organización, de esta manera se aseguraría en alguna medida un mejor desempeño laboral.

Al mismo tiempo la organización debe continuar aprovechando los beneficios que ofrece la gerencia de tecnologías de la información en el registro, procesamiento y difusión de la información de los procesos que se manejan internamente, manteniendo así los niveles de utilización los medios de producción de información, como lo son: bases de datos, página web, teleconferencias, correo electrónico, los cuales hasta ahora han alanzado excelentes resultados; $y$ el nivel de utilización de los elementos del diseño de proceso, la gestión del cliente, el desarrollo de los empleados, y la planificación del trabajo ya que son el soporte operativo de la organización.

\section{Conclusiones}

La revolución de la información se inició hace ya varios años, la atención de los actuales gerentes debe enfocarse en el desarrollo vertiginoso, constante y evidente de las TIC's, y en el cambio que el desarrollo tecnológico esta generando en esta sociedad del siglo XXI. La evolución acelerada de las TIC's ha generado cambios en todos los ámbitos sociales, económicos y financieros; ha producido la aparición de una cultura informática que está perturbando directa o indirectamente los valores morales y éticos de los individuos y de las organizaciones.

El presente papel de trabajo deja en evidencia que las organizaciones actuales se encuentran en una situación re- lativamente apremiante. Los gerentes de tecnología en cuyas decisiones está incorporar o no las tecnologías de información y comunicación a su organización, deben aceptar las potencialidades para el mejoramiento de los procesos organizacionales que ofrecen estas tecnologías.

Por otra parte, esta claro que para cualquier organización asumir el reto de pasar de la sociedad post-industrial o del entrenamiento a la sociedad de la información y del conocimiento, no es nada fácil. La incorporación de las TIC's en los procesos organizacionales trae consigo fuertes amenazas.

Las organizaciones inmersas en la sociedad de la información tienen preparada una artillería ética que permite defender y proteger de los ataques continuos de aquellos quienes pretenden vulnerar, entorpecer y dañar la privacidad. Las organizaciones realizar frecuentemente cambios en su estructura informacional para enfrentar la revolución de las TIC's, desarrollan nuevos modelos gerenciales y fortalecen de esa forma su capacidad productiva no sólo de información sino también del conocimiento.

Estos cambios generalmente producen dilemas de carácter ético, amenazas en el comportamiento y conducta de los individuos, de la sociedad y de la organización, han hecho crecer la desigualdad a favor de nuevos procesos de centralización de poder y concentración de riqueza, han creado nuevas barreras entre la gente de distinta cultura, estatus social y nivel de desarrollo, desigualdad en el acceso a la información.

Estos problemas implican mucho más que un análisis profundo de la ética informática y de la implantación de los có- 
digos de ética en el uso de las TICs. Las organizaciones actuales discuten sobre la posibilidad de formular proyecto de vida organizacional, fortaleciendo sus principios éticos, promulgando y estimulando el pensamiento moral y ético, todo esto con el único fin de constituirse en una organización vital donde lo más importante es la vida. La ética de la lógica de la vida les permitirá prepararse para enfrentar los retos de la guerra de la información en la era actual de la post-información.

\section{Referencias Bibliográficas}

Álvarez R., Víctor y Rodríguez Davgla (1998). "Hablemos .... De la Sociedad Rentista a la Sociedad del conocimiento". Fundacite-Zulia, Venezuela.

Brown, M.T. (1992). La ética en la empresa. Paidós, Barcelona.

Capurro, Rafael (2005). Ética de la Información. Un intento de ubicación. Traducción de un artículo en alemán publicado en la International Review of Information Ethics (1/2004). Fue publicada en la revista Códice : Revista de la Facultad de Sistemas de Información y Documentación. Universidad de la Salle (Bogotá, Colombia). Vol. 1, no. 2 (jul.-dic., 2005); p. 87-95 ISSN 1794-9815. [Documento en línea]. Disponible: http://www.capurro.de/colombia.htm. [Consulta 2006, marzo, 06]

Castells, Manuel (1999a). La Era de la Información. Economía, Sociedad y Cultura (Vol. I: La Sociedad Red); Siglo XXI Editores. 1era edición en español. Madrid, España.

Castells, Manuel (1999b). La Era de la Información. Economía, Sociedad y Cultura. (Vol. III: Fin de Milenio); Siglo
XXI Editores. 1era edición en español. Madrid, España.

Cornella, Alfons (1994). Los Recursos de Información, Ventaja competitiva de las empresas. Serie McGraw-Hill de Management. Madrid, España.

Cortina, Adela (1998). Ética de la Empresa. Tercera edición. Editorial Trotta. Madrid, España.

Del Brutto, Bibiana Apolonia (2003). Globalización y el nuevo orden internacional: las sociedades de la información, Revista TEXTOS de la CiberSociedad, 3. Temática Variada. Disponible en http://www.cibersociedad.net

Fernández, Carlos. Los Aspectos Éticos en la Formación de los Profesionales de la Información. Journal of Spanish Research on Information Science / Revista de Investigación Iberoamericana en Ciencia de la Información y Documentación. Vol. I / Nr. 2 Julio-Diciembre de 2000. Universidad de Granada (España). [Documento en línea]. Disponible: http://www.ucm.es/info/ multidoc/publicaciones/journal/pdf/ aspectos-eticos.pdf. [Consulta 2006, marzo, 04]

Floridi, Luciano (2000). Entropía como el Mal en las Ética de Información. Terza Università di Roma - University of Oxford. [Documento en línea]. Disponible: http://www. (http://www.wolfson. ox.ac.uk/ floridi/).. [Consulta 2005, febrero, 06]

Floridi, Luciano (2000). Mejora, modelos especulativos y búsquedas educativas en la multimedia de la interacción. Terza Università di Roma - University of Oxford [Documento en línea]. Disponible: (http://www.wolfson. ox.ac.uk/ floridi/) [Consulta 2005, febrero, 06]

Floridi, Luciano (2000). La Ética de la información: en la Fundación Filosófica de Ética de la Computadora. Terza 
Università di Roma - University of Oxford [Documento en línea]. Disponible: (http://www.wolfson.ox.ac.uk/ floridi/). [Consulta 2005, febrero, 06]

Hajna, Rifo, Lagreze, Byrt y Muñoz, Navarro (1989). Derecho e Informática, Ediciones Instituto profesional de Santiago. pp1-86.

Joyanes, Luis (1997). Cibersociedad. Los retos sociales ante el nuevo mundo digital. McGraw-Hill. Madrid, España.

MC. Leod, R. (2000). Sistemas de Información Gerencial. Pearson Education.

Moor, James $\mathrm{H}$. What is Computer Ethics, Metaphilosophy, Vol. 16, No. 4, October 1985, pp. 265-275.

OCDE (2002). Measuring the information economy. $p$ 81. HYPERLINK "http://www. oecd.org/" http://www. oecd.org/EN/ document/0EN-document-570-nodirectorate-no-135663-13,00.html

Parker, Donn, Swope, Susan y Baker, Bruce (1990). Ethical conflicts in Information and computer science, technology, and business. QED Information Sciences, Wellesley-USA.
Stein, George (2000). La Guerra de Información. Airpower Journal.

Silva, Neif (2001). La Gerencia de Tecnologías de Información y la Vinculación Universidad Sector Productivo. Trabajo de Tesis Doctoral. Programa de Doctorado en Ciencias Gerenciales. URBE.

Silva, Neif (2006). Cultura Informática en la Gestión de Actividades Académicas. En Memorias de la 5 Conferencia Iberoamericana en Sistemas, Cibernética e informática CISCI 2006. Volumen II. Ponencia. Orlando, Florida-EEUU.

Valls, Álvaro L.M. (2000). Que é Ética, Edit. Brasiliense, Coleção Primeiros Passos, no 177, 13aㅡ reimpressão, ano 2000, São Paulo.

Zavarce C., Carlos (1998). Gerenciando el Cambio Habilitado por la Tecnología de Información. Revista Educación y Ciencias Humanas. 